\title{
MODELLING AND SIMULATION OF QUALITY RISK FORECASTING IN A SUPPLY CHAIN
}

\author{
Liu, C. H. ${ }^{*} \&$ Xiong, W.* \\ *School of Business, Jiaxing University, Jiaxing, China \\ ${ }^{* *}$ School of Management, Zhejiang University, Hangzhou, China \\ E-Mail: liuch0001@163.com,wxiong@zju.edu.cn
}

\begin{abstract}
In recent years, the quality risks in supply chain are frequently encountered by Chinese's manufacturing enterprises, the aim of the paper is to present a manufacturer perspective methodology of forecasting quality risks in supply chain. To do it, firstly, we innovatively propose achieving factors of quality risks by the house of quality of quality function deployment. Secondly, an optimal selection approach regarding support vector machine parameters is suggested based on chaos particle swarm optimization, and then the forecasting model based on support vector machine is advanced. Finally, the experimental simulation of the model should be carried on some sample data. The results show that the forecasting accuracy and generalization ability of the proposed model is higher than particle swarm optimization-support vector machine on the same data sets. Therefore, the proposed method can be considered as a promising alternative method for forecasting quality risks in a supply chain from the perspective of manufacturers in China.

(Received, processed and accepted by the Chinese Representative Office.)
\end{abstract}

Key Words: Quality Risks, Chaos Particle Swarm Optimization, Support Vector Machines, Forecast Modelling, Supply Chain, House of Quality, Quality Function Deployment

\section{INTRODUCTION}

Under the dynamic environment, supply chain is subject to affected by all kinds of risks [1], including of strategic, tactical, organizational and business environment factors [2], which are finally shown in the form of quality risks. Enterprise, who only focuses on the internal quality risks management and ignores the quality risks from upstream and downstream, tends to encounter many quality problems of products or services [3]. To improve their global competitiveness by the means of risk management, the risk forecasting research is necessary. As we all know that it is all but is impossible to predict accurately. Even so, it is feasible to predict the risk patterns or trends for the short term from the perspective of macro management. So is our study goal. Our research will focus on three issues.

On one hand, for the risk factors of quality in supply chain are more complicated and more subtle, we are confronted with the challenge of finding quality risk factors clearly and objectively. According to the official definition of quality (namely ISO 8402,) given by international standard organism, which explains that quality is an inherent feature of products or services, namely quality is a kind of ability of meeting customer's requirements or expectations. Naturally, we think that the key of designing and managing quality is to transform the quality requirements into functional or measurable characteristics of quality [4, 5]. Moreover, the analysis and design tasks can be supported well by quality function deployment. So, we innovatively propose the method of analysing quality risk factors by house of quality of quality function deployment.

On the other hand, as for the method of risks forecasting, we usually classify it from these aspects. According to the study paradigm of prediction, forecast methods can be divided into qualitative and quantitative [6]. Quantitative forecasting is to estimates the future state of things (establish quantitative relations between models) by the quantitative techniques, such as predictive function. From the range of prediction, forecasts can be divided into macro 
forecast and micro forecast [7]. Macro forecast focus on the overall trend of a system risk. Micro forecast is aimed at the certain behaviours or local aspect. With regard to predicting technology, there is statistical method and artificial intelligent method [8]. At present, the artificial intelligence technology is more popular. Commonly neural networks, genetic algorithms, agents, ant algorithms and support vector machines, and so on are seen as valid artificial intelligence algorithms in forecasting. Some of these algorithms, such as neural network and genetic algorithms are often prone to "over fitting" [9, 10]. In contrast, support vector machines is not only a machine learning method of severing small sample, but also has structural risk minimization and strong generalization [11-13]. In recent years, support vector machines have been successfully applied in nonlinear system identification and prediction [14]. However, the forecasting accuracy based on small sample is relatively poor. In order to make up the defect of insufficient samples, we should adopt the global forecasting methods to extend the unknown information points. So, we put forward the combining method of chaos particle swarm optimization and support vector machine. Because the contribution of chaos to the diversity of particle and the iteration of particles [15], to some extent, the efficiency of algorithm and accuracy of support vector machines classifier can be improved.

Finally, in light of the complex of quality risks in supply chain, the forecast modelling on quality risks in supply chain will be carried on by this the combining method. The chaos particle swarm optimization algorithms are used in optimizing the parameters of radial basis functions for support vector machines. The presented forecasting models will apply in the typical quality risk prediction of supply chain, and the related simulation experiments are conducted to test the performance of the proposed model.

\section{OUALITY FUNCTION DEPLOYMENT}

\subsection{Analysis of quality risks in supply chain}

Because of the complexity of quality risks in supply chain and the limitations of disclosure, its definitions are diverse from the different perspective of researchers. But one thing is sure: the quality risks in supply chain are the sum of quality risks of all aspects in a supply chain [16]. And the quality risks refer to the probability of negative events caused by the conflict of quality requirements and quality reality. As a result, it maybe leads to a certain quality loss or other adverse consequences. According to the operation stages and management factors of supply chain, the risk of supply chain relates to the risks of production, sales, supply, management, overall quality, culture, and other aspects, in which quality risks in supply chain are the most critical. Most experts think that it is more difficult to prevent and manage the quality risks in supply chain. Compared with other risks in supply chain, these quality risks have some unique characteristics: transitivity, time lag, complexity, interaction and easily happening [17].

In fact, the quality risks are mainly reflected in the form of product quality risks and the service quality risks. Besides, the level of product and service is ultimately to be judged by customers, namely, the customer satisfaction to requirements is a measure of quality standards. Therefore, we take it into account that these quality risks, in fact, are caused by the conflicts between quality requirements and quality reality.

So, the requirements for supply chain quality (namely, voices of customers) should to be analysed, and gradually to be turned into the quality characteristics of supply chain. 


\subsection{Quality factors analysis based on quality function deployment}

The concept of quality function deployment

Quality function deployment tools emerged from Japanese design practice as means to integrate customer requirements and other lifecycle concerns into product design for improving its quality $[18,19]$. Its theoretical core is house of quality, which can be applied to decompose the customer requirements into the various stages of the whole process. The house structure of quality is shown as Fig. 1.

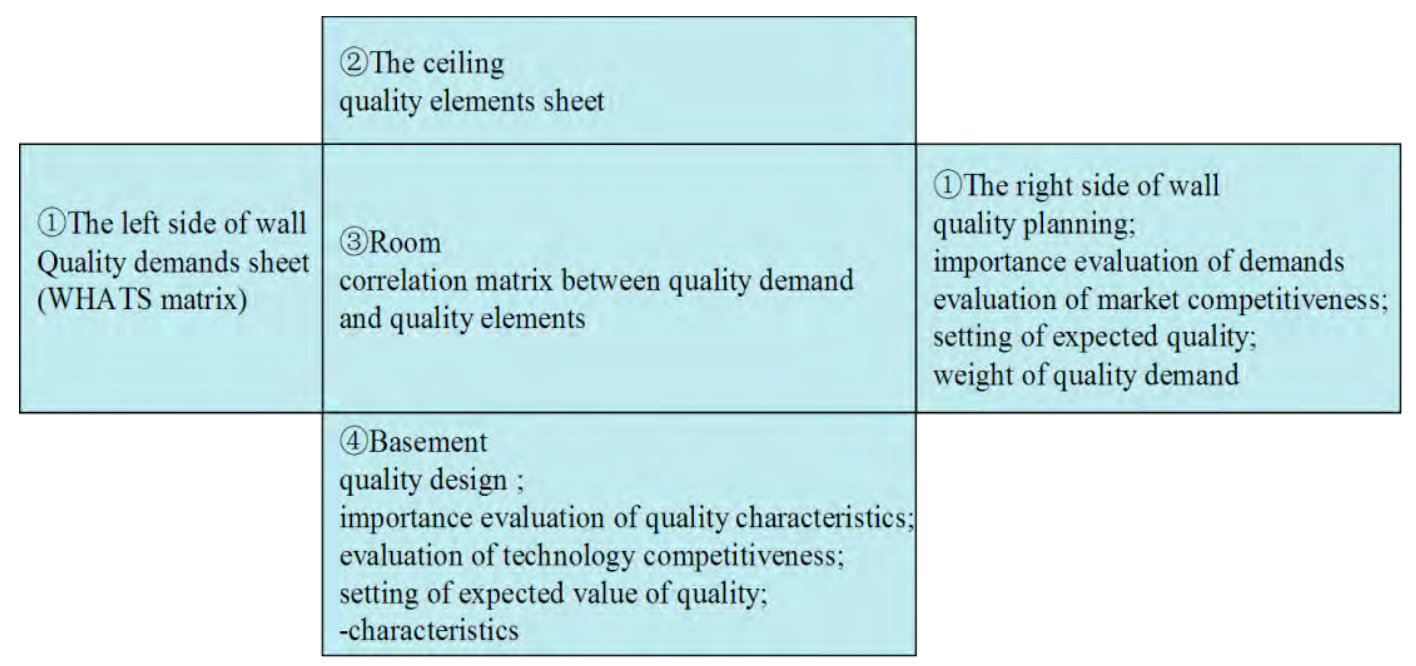

Figure 1: House structure of quality.

As shown in Fig. 1, the house of quality contains valuable information about the customer requirements and their relative importance, the relationships between the customer requirements and technical features of product quality, the correlation and the relative importance of features. We focuses on the matrix of house of quality, which can make us get the quality factors.

The house of quality of supply chain and the weights of quality risks factors

From above we can know that there is an organic link between the quality requirements and quality factors. Thankfully, quality function deployment can help us to do it. Its house of quality technology is a kind of means to correlate the customer requirements with the product quality or service performance.

On the basis of quality function deployment, we are to deploy the "voices of customers" into the quality assurance measures properties of supply chain, and the "quality properties of supply chain" separately by the house of quality of "customer demand to supply chain quality assurance measures characteristics" and the house of quality of "supply chain quality assurance measures characteristics to supply chain quality factors", which are shown in Fig. 2.

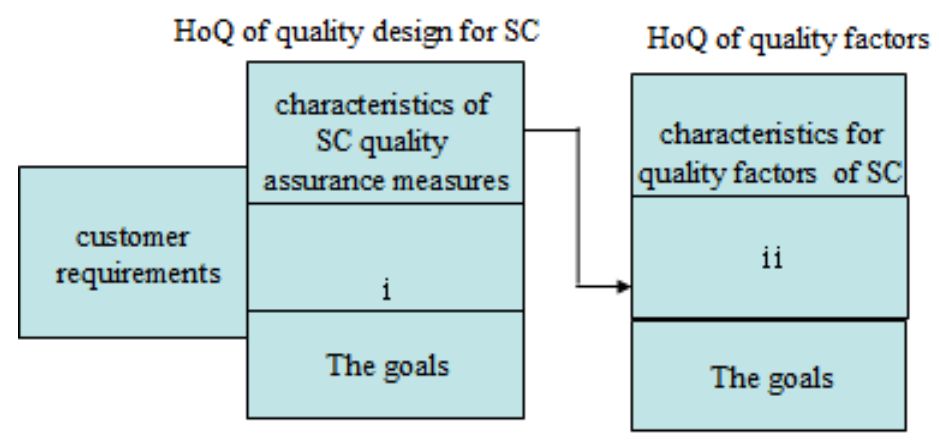

Figure 2: The deployment model of supply chain quality function. 
In tradition, the correlation between characteristics is given by experts. To avoid of the subjective solving of correlation and weights, we give relation value according to the survey questionnaire of the customers' requirements. For example, the strategic relationship as quality characteristic is just the embodiment of open cooperation needs, and then there is a correlation between both. The degree of association can be set to the level of feature meet to demand. The relevance between the demand of opening up and strategic cooperation relationship can be set according to the strategic cooperation relationship of the frequency within a certain time. In addition, to research easily, all relevance between requirements' properties and characteristics of quality will be set strong relevant, general relevant, weak correlation of three grades, which are respectively set as three values of 7,5 , and 3 .

Then, according to Fig. 2, the weights of parameters in house of quality can be calculated by the eqs. (1) and (2).

$$
\left.W_{R_{j}}=\left(\sum_{i=1}^{i=n} R_{j}^{i} / R_{j}^{Y}\right) * R_{j}^{Y} / \sum_{j=1}^{j=m} R_{j}^{Y}\right)
$$

The weights of output parameters are set by the eq. (2).

$$
\left.W_{B_{i}}=\left(\sum_{j=1}^{j=m} B_{i}^{j} / B_{i}^{Y}\right) * B_{i}^{Y} / \sum_{i=1}^{i=n} B_{i}^{Y}\right)
$$

where, $R$ is input vector of house of quality, such as customer requirements' parameters, $B$ is the output vector of house of quality, such as quality features' parameters, $W$ is the weights vector of parameters, $Y$ is the target values vector of parameters.

Logically, supply chain is actually a relational network to meet the supply and demand needs of all parties. The product quality and service quality are the main manifestation of supply chain quality, which result from all quality factors. So are the quality risks of supply chain. Based on this, the quality risk factors and their weights are studied as following.

The hierarchical model of quality risks in supply chain

Different from the traditional factor analysis by SPSS [20], the paper focuses on the customer's quality requirements to change these requirements into quality and technical characteristics by house of quality, and further converted into the characteristics of risk factors. So, according to the Fig. 2, the hierarchical model of quality risk factors in supply chain is shown as Fig. 3.

On account of these quality risks factors in Fig. 3 play force in the order of supply chain system, we propose the information entropy to express this force. Thus, the eqs. (3) and (4) are designed to calculate the information entropy of risk factors in Fig. 3.

Some steps should be taken to calculate the information entropy of indexes in fourth layer by eq. (4), then the information entropy of indexes in third layer and in second layer are solved by eq. (3), which can be used as control parameters for risk.

$$
\begin{gathered}
E_{U_{i j}}=\left(W_{U_{\mathrm{ij} 1}} * E_{U_{i j 1}}+W_{U_{\mathrm{ij} 2}} * E_{U_{i j 2}}+\ldots . W_{U_{\mathrm{ijk}}} * E_{U_{i j k}}\right) / k \\
E_{U_{i j k}}=\sum_{k=1}^{k=n^{\prime}} P_{k} * \log _{2}^{P_{k}}
\end{gathered}
$$

where $U_{i j}$ is the $j$ index in layer $i$, and then $W_{U_{i j 1}}$ presents the weight of $U_{i j}, E_{U i j}$ is regarded as the entropy of $U_{i j}, k$ is the total numbers of indexes in second layer, $E_{U i j k}$ presents the entropy of $U_{i j k}$, and $n^{\prime}$ is the total numbers of attributers for $k$ index. $P_{k}$ is seen as the information probability of $U_{i j k}$.

Based on the above analysis, according to the complexity and evolution of quality risks, the forecasting model of quality risks in supply chain will be constructed as following. 


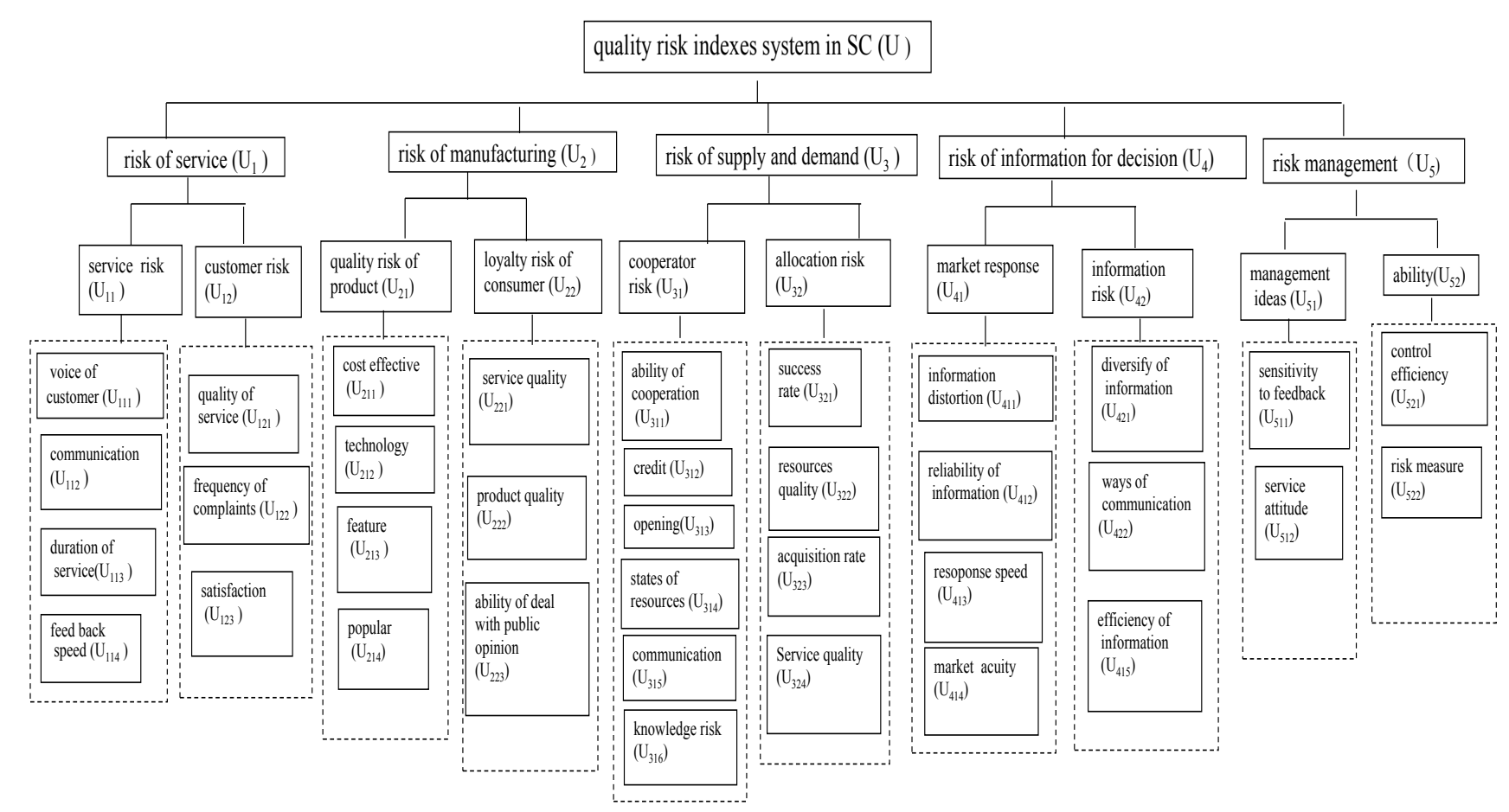

Figure 3: The hierarchical model of quality risks in supply chain.

\section{MODELLING PROCESSES}

\subsection{Review of chaos particle swarm optimization-support vector machines}

The basic idea of support vector machines is that for the non-linear separable samples, its input vector is mapped to a high-dimensional space to find an optimal interface (hyper plane) for promoting the performance of its search, and then obtaining classification [21].

Actually in the high-dimensional space, only inner product operation is required, which can be implemented through the function of original space, as long as the kernel function is fit to the Mercer condition. As a result, operations based on high-dimension can be realized through a lower-dimensional operation nonlinearly.

Generally, support vector machines and neural networks can be used to make non-linear regression fit, but they are not the same principle. Support vector machines based on structural risk minimization theory, can be obsessed stronger generalization than neural network. However, it is very difficult to choose a kernel function and its parameter values for the modelling of support vector machines, whose performance depends on the appropriate values of parameters [22].

As for kernel functions, there are commonly linear kernel function, a polynomial kernel function, sigmoid kernel and radial basis kernel function which, compared to other kernels functions, has fewer parameters and good performance[23, 24]. So, the radial basis kernel function will be used in this study.

As we know that under the insufficient historical data, the global forecasting method should to be required. Because it is difficult to obtain the data of quality risks in supply chain from statistical tables, we present to extract these sample data from the risk cases occurred in some enterprise. Taking into account the small size of sample data, the global prediction method based on support vector machines built on chaos particle swarm optimization should be used to improve the convergence speed and accuracy of support vector machines. Chaos based particle swarm optimization can be help in improving the iteration and diversity of particle swarm, so that the chaos particle swarm optimization is avoid of the local maximum 
point [25]. Therefore, this Particle Swarm based on chaos evolution is used to determine the optimum parameters for radial basis function of support vector machines.

Here, through the above description of chaos particle swarm optimization based support vector machines, we aim to create the model of forecasting quality risks in supply chain of manufacturing enterprise.

\subsection{Modeling thoughts of forecasting quality risks in supply chain for manufacturers}

In order to convenient modelling, we put forward the assumption: do not consider the natural factors of risks and political factors of risks, according to the customers' orientated analysis the quality risk factors in this paper are related to product quality and service quality.

In this research, the radial basis function is taken as the classifiers of support vector machines, so the kernel parameter $\sigma$ and the penalty parameter $C$ are two indispensable parameters. To find the optimal values of parameters, the global search operation is run through the chaos particle swarm. And then, the chaos particle swarm optimization based support vector machines model will be set by the following steps.

(1) The regression equation of support vector machines

Support vector machine as a machine learning algorithm is commonly used to provide the best generalization by seeking the best compromise between model complexities and learning ability [26]. Assuming there is only a pair of input and output $(x(n), y(n))$, the regression function of SVR can be expressed as:

$$
f(x)=w \times \varphi(x)+b
$$

In eq. (5), $\varphi(x)$ stands for the nonlinear mapping function from the input space to the highdimensional space of features. In addition, $w$ with independence represents the weight vector of the input vector which can be gained by eqs. (6) and (7), $b$ says the bias vector.

Because of its main idea is to build a hyper plane for classify, so, it is equivalent to solving the following optimization problem:

$$
\min \frac{1}{2}(w \cdot w)+C \sum_{i=1}^{l} \xi_{i}
$$

with constraints:

$$
\begin{aligned}
& y_{i}\left(\left(w \cdot x_{i}\right)+b\right) \geq 1-\xi_{i}, i=1, \ldots, l \\
& \xi_{i} \geq 0, i=1, \ldots, l
\end{aligned}
$$

where, the symbol of $\xi_{i}$ is a slack variable giving a soft classifying boundary. The symbol of $C$ is a punishment constant.

The Lagrange multipliers $a_{i}$ and $a_{i}{ }^{*}$ are introduced into constructing a Lagrange function. Then, the support vector machines decision function can be carried out as follows:

$$
f(x)=\sum_{i=1}^{l}\left(a_{i}-a_{i}^{*}\right) K\left(x_{i}, x\right)+b
$$

with constraints:

$$
0 \leq a_{i} \leq C, i=1, \ldots, l
$$

where, kernel function $K\left(x_{i}, x_{j}\right)$ is used to transform the original input space to a higher dimensional feature space.

Based on the radial basis function, the regression function of support vector machines is taken on as following:

in which, $\sigma$ is the width of kernel basis function.

$$
f(x)=\sum_{i=1}^{1} a_{i} * \exp \left(\frac{\llbracket x_{i}-x_{i} \rrbracket^{2}}{2 \sigma^{2}}\right)+b
$$


(2) The algorithm ideas of chaos particle swarm optimization

Although support vector machines can effectively solve the problem of small sample, nonlinear, high dimension and local minima problems, for the performance of support vector machines depends closely on its parameters, the support vector machines parameter selecting (including the selection of the kernel function parameters) is an important issue in continuous improvement [27]. Therefore, the chaos particle swarm optimization algorithm is used to optimize parameters of support vector machines.

Step 1: Particle swarm optimization is initialized with a swarm including $N$ random particles. Each particle is treated as a point in a $D$-dimensional space. The $i^{\text {th }}$ particle is represented as $x_{i}=\left(x_{i 1}, \ldots, x_{i D}\right), x_{i j}$ is limited in the range $\left[a_{j}, b_{j}\right]$. The best previous position of the $i^{\text {th }}$ particle is represented as $P_{i}=\left(p_{i 1}, \ldots, p_{i D}\right)$. The best particle among all the particles in the population is represented by $P_{g}=\left(p_{g 1}, \ldots, p_{g D}\right)$. The velocity of particle $i$ is represented as $V_{i}=\left(v_{i 1}, \ldots, v_{i D}\right)$. After finding the aforementioned two best values, the particle updates its velocity and position according to the following equations [28, 29]:

$$
\begin{gathered}
v_{i d}=w v_{i d}+C R\left(p_{i d}-x_{i d}\right)+c r\left(p_{g d}-x_{i d}\right) \\
x_{i d}=x_{i d}+v_{i d}
\end{gathered}
$$

where, $d$ is the $d^{\text {th }}$ dimension of a particle, $w$ is the inertia weight, $C$ and $c$ are two positive constants called learning factors, $R$ and $r$ are random numbers in the range of $[0,1]$.

Step 2: Because chaos queues can experience all the states in a specific area without repeat, chaotic search becomes a novel tool used as an optimizer. Here, Logistic equation is employed to obtain chaos queues, which is represented as follows:

$$
x_{n+1}=u * x_{n}\left(1-x_{n}\right), \quad x_{n}=\left(x_{11}, x_{12}, \ldots x_{1 n}\right)
$$

where, $u$ is the control parameter, suppose that $0 \leq x_{n} \leq 1, n$ represents the number of dimensions of the vector $x_{n}$.

(3) The algorithm ideas of chaos particle swarm optimization

The basic idea of chaos particle swarm optimization is described as follows:

Step 1: Sample collection and data normalization.

Step 2: Construction of the training sample set.

Step 3: Training normalized samples to obtain the optimal parameters of support vector machines.

Step 4: According to the optimal parameter values to establish the chaos particle swarm optimization-support vector machines based model, and then by the anti-normalization of test results we was obtained the forecast resulting.

\section{SIMULATION PROCESSES AND RESULTS}

\subsection{Simulation environment}

To test the performance of the quality risk prediction model for supply chain. The proposed algorithm has been developed using the MATLAB platform version 7.10.0 (MATLAB version 7.10.0 (R2010a)). The sample data for simulation are grasped from some risks' events occurred in manufacturing industries of China. The classification error for simulation is set as:

$$
1-\frac{1}{L} \sum_{i=1}^{L}\left|\frac{T_{i}}{F_{i}+T_{i}}\right| \times 100 \%
$$

where, $T$ is the correct classification, $F$ presents the misclassification. 


\subsection{Formation of the sample dataset}

A dataset collected from cases of four industries is established to train chaos particle swarm optimization-support vector machines and predict quality risks in supply chain.

The industries are classified into four kinds, such as the food and milk manufacturers (which indicates as $\mathrm{S}$ character), drug manufacturers $(\mathrm{Y})$, electronics manufacturers (D), and Chemical manufacturers $(\mathrm{H})$. The cases from these companies in recent years are selected as the analysis objects. In this study, we have acquired the information and data from a number of industry website (such as, http://www.chemchem.com.cn/, http://news.cecb2b.com/, http://news.iresearch.cn/, http://www.auto-china.cn/, http://www.jhyjj.cn/), the State Quality Supervision Bureau website (http://www.aqsiq.gov.cn/), other trusted websites and news reports in previous years. Moreover, according to the quality risk factors shown in Fig. 3, the source of sample data is collected and the entropies of risk indexes are computed by eqs. (3) and (4). Particularly, the entropies of indexes in the $3^{\text {rd }}$ level of Fig. 3 are turned into the initial values of $x$ particle in model of support vector machines. That is, the sample data processed is shown in Table I.

Table I: Training and testing dataset.

\begin{tabular}{|c|c|c|c|c|c|c|c|c|c|c|}
\hline \multirow[b]{2}{*}{ firms } & \multirow[b]{2}{*}{$\mathbf{I}_{1}$} & \multicolumn{9}{|c|}{$3^{r d}$ level inderes of risk madel } \\
\hline & & $\mathbf{I}_{2}$ & $I_{3}$ & $\mathbf{I}_{4}$ & $I_{5}$ & $\mathbf{I}_{6}$ & $\mathbf{I}_{7}$ & $\mathbf{I}_{8}$ & $\mathbf{I}_{9}$ & $\mathbf{I}_{10}$ \\
\hline$S_{1}$ & 0.4786 & 0.2291 & 0.8783 & 0.6879 & 0.1101 & 0.0120 & 0.3101 & 0.8971 & 0.1349 & 0.1490 \\
\hline$S_{2}$ & 0.8683 & 0.6591 & 0,7388 & 0.4503 & 0.7495 & 0.7990 & 0.0385 & 0.5676 & 0.0929 & 0.9811 \\
\hline $\begin{array}{l}S_{3} \\
S_{4}\end{array}$ & 0.1115 & 0.4935 & 0.0609 & 0.2798 & 0.5168 & 0.7867 & 0.6909 & 0.4674 & 0.0204 & 0.9070 \\
\hline$S_{5}$ & 0.6367 & 0.0123 & 0.7356 & 0.6979 & 0.2086 & 0.9423 & 0.8787 & 0.1584 & 0.3670 & 0.2792 \\
\hline$S_{6}$ & 0.2607 & 0.2534 & 0.3472 & 0.0682 & 0.0523 & 0.0487 & 0.3418 & 0.5329 & 0.5478 & 0.2002 \\
\hline$Y_{1}$ & 0.3436 & 0.9751 & 0.1359 & 0.0994 & 0.9626 & 0.1242 & 0.9140 & 0.7338 & 0.1658 & 0.4879 \\
\hline $\mathrm{Y}_{2}$ & 0,1805 & 0.8994 & 0.1540 & 0.3673 & 0.9666 & 0.1765 & 0.3594 & 0.1712 & 0.0479 & 0.9604 \\
\hline $\begin{array}{l}Y_{3} \\
Y_{4}\end{array}$ & 0.3484 & 0.1506 & 0.5211 & 0.2630 & 0.0305 & 0.9016 & 0.3695 & 0.5198 & 0.7209 & 0.2260 \\
\hline$Y_{5}$ & 0.2225 & 0.9292 & 0.6645 & 0.2607 & 0.2483 & 0.6364 & 0.8613 & 0.0417 & 0.6398 & 0.6895 \\
\hline$Y_{6}$ & 0.8280 & 0.1609 & 0.7170 & 0.6929 & 0.6901 & 0.3599 & 0.6544 & 0.5593 & 0.5654 & 0.8105 \\
\hline$D_{1}$ & 0.7494 & 0.9038 & 0.1394 & 0.5509 & 0.6688 & 0.1554 & 0.9444 & 0.1340 & 0.3078 & 0.1307 \\
\hline $\mathrm{D}_{2}$ & 0.3627 & 0.5169 & 0.0720 & 0.3027 & 0.0379 & 0.2502 & 0.7232 & 0.4862 & 0.6705 & 0.8255 \\
\hline $\begin{array}{l}D_{3} \\
D_{4}\end{array}$ & 0.9488 & 0.8933 & 0.1949 & 0.7114 & 0.5727 & 0.3335 & 0.1305 & 0.8126 & 0.4491 & 0.0460 \\
\hline$D_{5}$ & 0.1181 & 0.5881 & 0.3030 & 0.2279 & 0.2789 & 0.0332 & 0.5535 & 0.2970 & 0.8474 & 0.8890 \\
\hline$D_{6}$ & 0.2841 & 0.1481 & 0.7378 & 0,5406 & 0.3151 & 0.5944 & 0.9825 & 0.8508 & 0.1940 & 0.9574 \\
\hline $\mathrm{H}_{1}$ & 0.6017 & 0.7507 & 0.0327 & 0.5036 & 0.5865 & 0.3873 & 0.2346 & 0.7930 & 0.3265 & 0.7181 \\
\hline $\mathrm{H}_{2}$ & 0.6364 & 0.9151 & 0.2793 & 0,7449 & 0.4799 & 0.3438 & 0.0801 & 0.4802 & 0.7324 & 0.3602 \\
\hline $\begin{array}{l}\mathrm{H}_{3} \\
\mathrm{H}_{4}\end{array}$ & 0.3983 & 0.0497 & 0.5953 & 0.9483 & 0.5309 & 0.3151 & 0.2827 & 0.1590 & 0.9906 & 0.0950 \\
\hline $\mathrm{H}_{5}$ & 0.6948 & 0.1271 & 0.6842 & 0.4127 & 0.3849 & 0.0880 & 0.1129 & 0.3773 & 0.8060 & 0.5812 \\
\hline $\mathrm{H}_{6}$ & 0.0285 & 0.4674 & 0.9715 & 0.1474 & 0.4413 & 0.8738 & 0.5598 & 0.7032 & $0.016 T$ & 0.6156 \\
\hline
\end{tabular}

Nearly two-thirds of the sample data is set as a training sample set, and the rest is as a test sample.

\subsection{Chaos particle swarm optimization-support vector machines based forecast modelling and experimental simulation}

The parameters of chaos particle swarm optimization are set as follows: the size of the population $M=30$, the inertia weight $w$ decreases linearly from 1 to 0.4 , the optimal parameters of support vector machines searched by chaos particle swarm optimization are $\mathrm{C}=4.6428, \sigma=3.7863 \mathrm{e}-004$. For the sake of comparison, the optimal parameters of support vector machines searched by particle swarm optimization are $\mathrm{C}=4.1555, \sigma=0.81709$. At the 
same time, using the parameters determined by chaos particle swarm optimization to establish risk prediction models, the results of classification prediction are shown as Fig. 4.

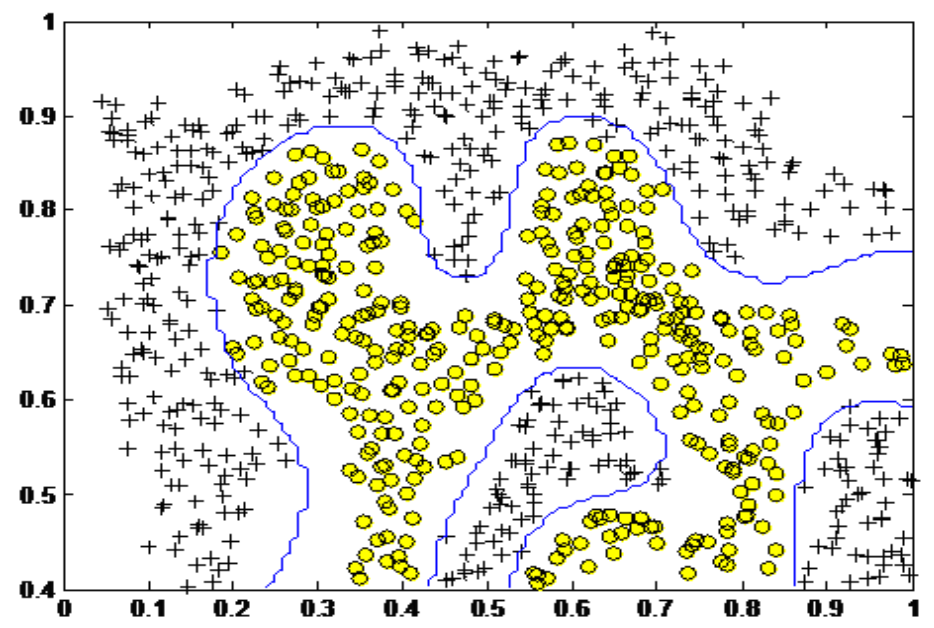

Figure 4: The boundary determination of support vector machines and risk prediction.

This is a map of particles motion. As we can be seen, the classification ability of this model is strong. The model reaches on the curve classification which is superior to the general linear classification. At the same time, in the prediction process of classification, few heterogeneous particles fall into the boundary, so that the training error is relatively small. It indicates the chaos particle swarm optimization-support vector machines algorithm with high precision.

To further validate the feasibility of this proposed model, to be compared with the support vector machines and particle swarm optimization based support vector machines algorithm, their forecasting results are shown as Fig. 5.

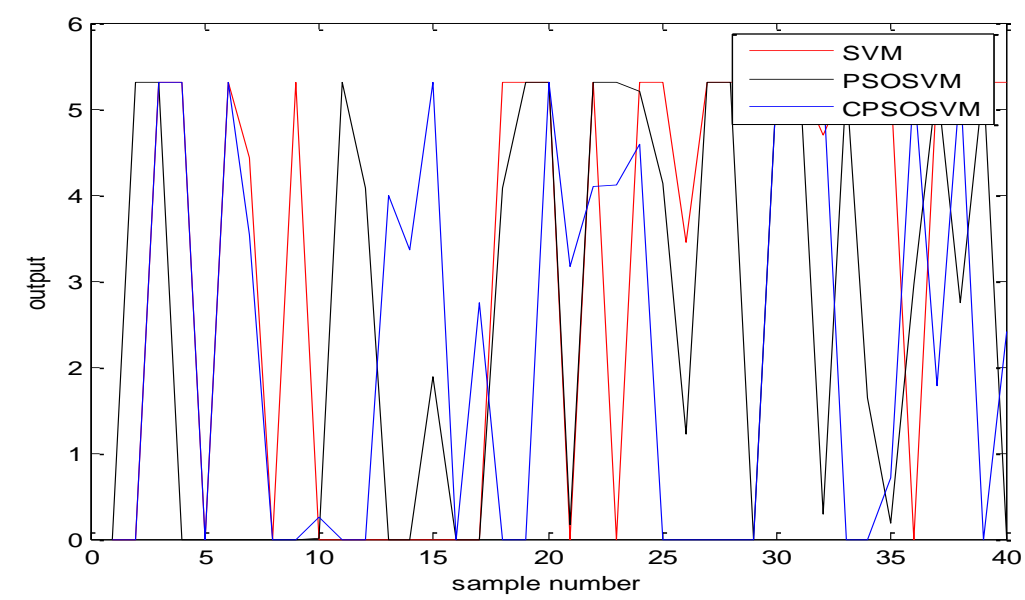

Figure 5: Comparison of prediction results.

The results can be seen in Fig. 5, compared to particle swarm optimization based support vector machines and support vector machines, when the sample data is very small, the chaos particle swarm optimization-support vector machines prediction accuracy is still high. In the whole process, chaos particle swarm optimization based support vector machines is not fall into local optimum. It displays that prediction accuracy of chaos particle swarm optimization based support vector machines model is better than particle swarm optimization-support vector machines and support vector machines.

Specifically, the miss rate of experimental results and prediction accuracy are given in Table II. 
Table II: Comparison of prediction accuracy.

\begin{tabular}{|l|c|c|c|c|c|c|}
\hline \multicolumn{1}{|c|}{ Algorithms } & $\begin{array}{c}\text { Number } \\
\text { of points }\end{array}$ & Miss rate & Weight 1 & Weight 2 & $\boldsymbol{b}$ & $\begin{array}{c}\text { Prediction } \\
\text { accuracy (\%) }\end{array}$ \\
\hline Support vector machines & 227 & 19 & -0.5589 & 1.5535 & 0.4087 & 91.63 \\
\hline $\begin{array}{l}\text { Support vector machines } \\
\text { based on particle swarm } \\
\text { optimization }\end{array}$ & 147 & 10 & -0.5137 & 2.1593 & 0.4976 & 93.20 \\
\hline $\begin{array}{l}\text { Support vector machines } \\
\text { based on chaos particle } \\
\text { swarm optimization }\end{array}$ & 144 & 6 & -0.6036 & 2.2823 & 0.5084 & 95.83 \\
\hline
\end{tabular}

It is obvious that chaos particle swarm optimization-support vector machines algorithm has better prediction accuracy and stronger stability than that of support vector machines and particle swarm optimization-support vector machines. If more data were applied for particle swarm optimization based support vector machines training, particle swarm optimizationsupport vector machines will also have a greater generalization ability and study ability, but this improvement will consume more energy and time, in that sense, chaos particle swarm optimization based support vector machines has certain advantages that it can enrich data information by the evolution of only a small set of training data.

Besides, the convergence of chaos particle swarm optimization algorithm is superior to the particle swarm optimization algorithm. The comparison of convergence under different evolution environment by chaos particle swarm optimization is shown as Table III.

Table III: Comparison of convergence steps.

\begin{tabular}{|l|c|c|c|c|c|}
\hline \multicolumn{1}{|c|}{ Algorithms } & $\begin{array}{c}\text { Chaos } \\
\text { coefficient }\end{array}$ & $\begin{array}{c}\text { Population } \\
\text { size }\end{array}$ & $\begin{array}{c}\text { Evolution } \\
\text { times }\end{array}$ & $\begin{array}{c}\text { Particle } \\
\text { dimension }\end{array}$ & $\begin{array}{c}\text { Convergence } \\
\text { steps }\end{array}$ \\
\hline Chaos particle swarm optimization & 2 & 20 & 1000 & 10 & 112 \\
\hline Chaos particle swarm optimization & 1.5 & 20 & 1000 & 10 & 98 \\
\hline Chaos particle swarm optimization & 3 & 20 & 1000 & 10 & 101 \\
\hline Particle swarm optimization & $\ldots$ & 20 & 1000 & 10 & 950 \\
\hline Particle swarm optimization & $\ldots$ & 16 & 1000 & 10 & 960 \\
\hline Particle swarm optimization & $\ldots$ & 13 & 1000 & 10 & 980 \\
\hline
\end{tabular}

It can be seen from Table III that particle swarm optimization with chaos coefficients has strong optimization, and can reach the target error only by 100 steps. On the contrary, it will consume 950 steps for particle swarm optimization.

Therefore, regardless of the predict precision and the algorithms convergence, the chaos particle swarm optimization is better than particle swarm optimization.

\section{CONCLUSIONS}

In this study, we applied support vector machines optimized by chaos particle swarm optimization into quality risks forecasting of supply chain. Chaos particle swarm optimization primarily achieved parameters of $C$ and $\sigma$ to build an effective forecasting model. Before this, we also should provide the risk index system by house of quality of quality function deployment, so as to calculate the weights and entropies on risk factors and risk indexes. We take each risk factor as a sub-particle swarm consisted of its indexes-particles. Thereby, the risk factors are regarded as chaotic variables, and make entropies of risks as the input $w$ values to $x$ vector. Results of simulation show that the model proposed overcomes the main shortage of easily trapping in the local optimum for a traditional support vector machines. 
Compared with particle swarm optimization-support vector machines and support vector machines, a chaos particle swarm optimization-support vector machine has better performance.

The simulation on some risk cases presents the forecasting model has certain help in quality risk prediction of supply chain. In addition, this model has the possibility for application in other risk forecast fields, especially which possess nonlinear evolution with small sample data.

\section{ACKNOWLEDGEMENTS}

This work is supported by the natural science foundation of Zhejiang province in China (Grant No. LY13G010005), supported by the major program of the National Social Science Foundation (Grant No. 12 \& ZD206) and supported by the postdoctoral foundation of China (Grant No. 2013M541792).

\section{REFERENCES}

[1] Wang, Y.-M.; Zhao, D.-Z.; Huang, J. (2009). The supply quality cost control model based on risk transmission, Science and Technology Management Research, Vol. 28, No. 3, 155-157

[2] Denic, N.; Moracanin, V.; Milic, M.; Nesic, Z. (2014). Risk management in information system projects, Technical Gazette, Vol. 21, No. 6, 1239-1242

[3] Tang, M. (2011). Research on quality warranty contract in supply chain considering moralhazard, Master's Thesis, Nanjing University of Aeronautics and Astronautics, China

[4] De Cerio, J. M. (2003). Quality management practices and operational performance: Empirical evidence for Spanish industry, International Journal of Production Research, Vol. 41, No. 12, 2763-2786, doi:10.1080/0020754031000093150

[5] Flynn, B. B.; Schroeder, R. G.; Sakakibara, S. (1995). The impact of quality management practices on performance and competitive advantage, Decision Sciences, Vol. 26, No. 5, 659-691, doi:10.1111/j.1540-5915.1995.tb01445.x

[6] Fjodorova, N.; Vračko, M.; Tušar, M.; Jezierska, A.; Novič, M.; Kuhne, R.; Schuurmann, G. (2010). Quantitative and qualitative models for carcinogenicity prediction for non-congeneric chemicals using CP ANN method for regulatory uses, Molecular Diversity, Vol. 14, No. 3, 581594, doi:10.1007/s11030-009-9190-4

[7] Vargas, R. O.; Manero, O.; Phillips, T. N. (2009). Viscoelastic flow past confined objects using a micro-macro approach, Rheologica Acta, Vol. 48, No. 4, 373-395, doi:10.1007/s00397-0080323-y

[8] Kumar, P. R.; Ravi, V. (2007). Bankruptcy prediction in banks and firms via statistical and intelligent techniques - A review, European Journal of Operational Research, Vol. 180, No. 1, 1-28, doi:10.1016/j.ejor.2006.08.043

[9] Wang, Y.; Chen, C.; Yan, X.-F. (2013). Structure and weight optimization of neural network based on CPA-MLR and its application in naphtha dry point soft sensor, Neural Computing and Applications, Vol. 22, No. 1, Supplement, 75-82, doi:10.1007/s00521-012-1044-9

[10] Jimenez, A.; Beltran, G.; Aguilera, M. P.; Uceda, M. (2008). A sensor-software based on artificial neural network for the optimization of olive oil elaboration process, Sensors and Actuators B: Chemical, Vol. 129, No. 2, 985-990, doi:10.1016/j.snb.2007.09.030

[11] Sun, L.; Wang, D.-Y. (2012). Optimal structural design of the midship of a VLCC based on the strategy integrating SVM and GA, Journal of Marine Science and Application, Vol. 11, No. 1, 59-67, doi:10.1007/s11804-012-1106-5

[12] Li, W.-H.; Liu, L.-J.; Gong, W. (2011). Multi-objective uniform design as a SVM model selection tool for face recognition, Expert Systems with Applications, Vol. 38, No. 6, 6689-6695, doi:10.1016/j.eswa.2010.11.066

[13] Liu, X.; Lu, W.-C.; Jin, S.-L.; Li, Y.-W.; Chen, N.-Y. (2006). Support vector regression applied to materials optimization of sialon ceramics, Chemometrics and Intelligent Laboratory Systems, Vol. 82, No. 1-2, 8-14, doi:10.1016/j.chemolab.2005.08.011 
[14] Hwang, S. H.; Ham, D. H.; Kim, J. H. (2012). Forecasting performance of LS-SVM for nonlinear hydrological time series, KSCE Journal of Civil Engineering, Vol. 16, No. 5, 870-882, doi:10.1007/s12205-012-1519-3

[15] Mahdiyeh, E.; Hussain, S.; Azah, M. (2011). Power system stabilizer design using hybrid multiobjective particle swarm optimization with chaos, Journal of Central South University of Technology, Vol. 18, No. 5,1579-1588, doi:10.1007/s11771-011-0875-3

[16] Wang, L.-N.; Lu, L.-L. (2012). Research on coordination in supply chain for perishable goods based on quality risk, Luo, J. (Ed.), Soft Computing in Information Communication Technology, Vol. 161, Springer-Verlag, Berlin, 501-509, doi:10.1007/978-3-642-29452-5 71

[17] Jiang, J.-D.; Zhao, H.-P.; Feng, Y.-C. (2008). Study on the features of quality risk in supply chain, Aeronautic Standardization \& Quality, Vol. 23, No. 1, 29-33, doi:10.13237/j.cnki. asq.2008.01.014

[18] Akao, Y. (Ed.), (1990). Quality Function Deployment: Integrating Customer Requirements into Product Design, Productivity Press, Cambridge (original Japanese version from 1988)

[19] Prasad, B. (1998). Review of QFD and related deployment techniques, Journal of Manufacturing Systems, Vol. 17, No. 3, 221-234, doi:10.1016/S0278-6125(98)80063-0

[20] Wei, L.-Y.; Luo, Q.-L.; Huang, Q.-H. (2012). Research on teacher's teaching ability lack in independent college and countermeasures, China Electric Power Education, Vol. 34, 114-115

[21] Cristianini, N.; Scholkopf, B. (2002). Support vector machines and kernel methods: The new generation of learning machines, Ai Magazine, Vol. 23, No. 3, 31-41

[22] Bian, Z.-Q.; Zhang, X.-G. (2000). Pattern recognition, Tsinghua Press. Beijing (in Chinese)

[23] Dioşan, L.; Rogozan, A.; Pecuchet, J.-P. (2012). Improving classification performance of Support Vector Machine by genetically optimizing kernel shape and hyper-parameters, Applied Intelligence, Vol. 36, No. 2, 280-294, doi:10.1007/s10489-010-0260-1

[24] Phienthrakul, T.; Kijsirikul, B. (2010). Evolutionary strategies for hyperparameters of support vector machines based on multi-scale radial basis function kernels, Soft Computing, Vol. 14, No. 7, 681-699, doi:10.1007/s00500-009-0458-5

[25] Huang, J.; Bo, Y.-C.; Wang, H.-Y. (2011). Flue gas turbine state forecasting by chaos particle swarm optimization and SVR, Computer Simulation, Vol. 28, No.5, 193-196

[26] Zhao, Y.; Xiao, W.; Chen, A-L. (2012). Network traffic forecast based on weighted support vector regression, Computer Engineering and Applications, Vol. 48, No. 21, 103-106

[27] Su, G.-L.; Deng, F.-P. (2006). Introduction to model selection of SVM regression, Bulletin of Science and Technology, Vol. 22, No. 2, 154-158, doi:10.13774/j.cnki.kjtb.2006.02.004

[28] Tang, X.-L.; Zhuang, L.; Gao, Y.-H. (2011). Support vector machine based on chaos particle swarm optimization for lightning prediction, Jin, D.; Lin, S. (Eds.), Advances in Computer Science, Intelligent System and Environment, Vol. 104, Springer-Verlag, Berlin, 727-733, doi:10.1007/978-3-642-23777-5_117

[29] Zhu, F.-M.; Fan, M.-L. (2010). Chaos particle swarm optimization algorithm for optimizing the parameter of SVM, Computer Simulation, Vol. 27, No. 11, 183-186 